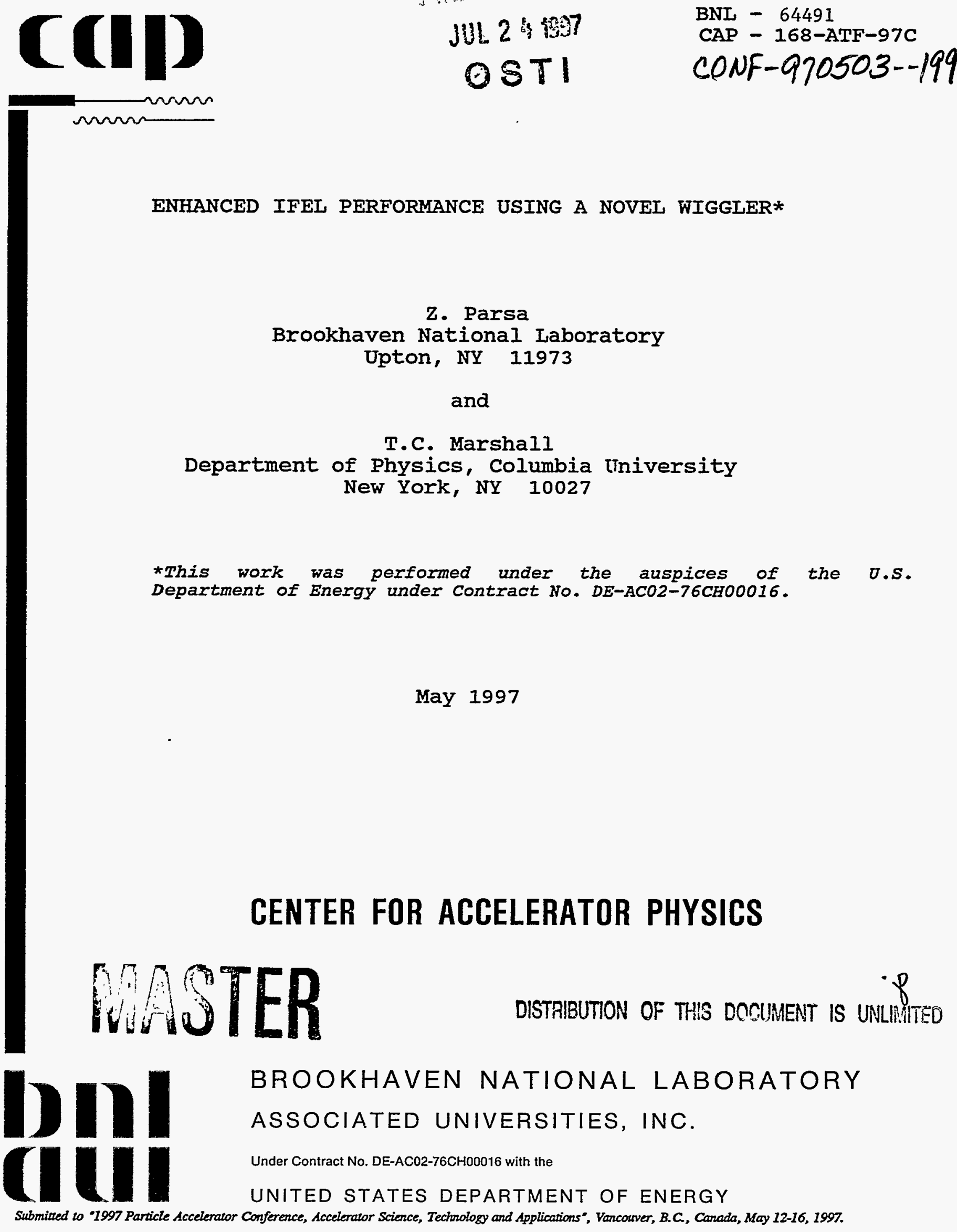




\section{DISCLAMIER}

Portions of this doecument may be illegible in electronic image products. Images are produced from the best available original document. 


\title{
ENHANCED IFEL PERFORMANCE USING A NOVEL WIGGLER
}

\author{
Z. Parsa \\ Brookhaven National Laboratory, Upton NY 11973 \\ T.C. Marshall \\ Department of Applied Physics, Columbia University, New York City 10027
}

\begin{abstract}
In the conventional inverse free electron laser (IFEL), electron acceleration is done via the interaction of an intense laser beam and a wiggler with a sinusoidal field variation. We have studied the effect on IFEL performance using a wiggler that creates a nearly squarewave periodic field pattern $[1,2]$. A novel numerical techniquie is described which models this wiggler [1]. We find stable orbits and an improvement in IFEL acceleration gradient, resulting in a gain in energy by as much as a factor of two (equivalent to four times the laser power), when compared with the conventional IFEL with a sinusoidal field wiggler [3]. In an experiment, the magnetic field can be synthesized by running a conventional ferromagnetic wiggler into saturation.
\end{abstract}

\section{INTRODUCTION}

The inverse FEL (IFEL) uses the principal of stimulated absorption to accelerate a beam of electrons passing through a wiggler using a source of high intensity electromagnetic waves. The first IFEL experiment was reported in 1991 using a $750 \mathrm{kV}$ electron beam that was prebunched by, and powered by, a millimeter-wavelength FEL [4]. Recently, acceleration of a $40 \mathrm{MeV}$ beam to $42 \mathrm{MeV}$ was reported [5] using a GW $\mathrm{CO} 2$ laser beam; the bunching of this beam is being studied [6] preparatory to injecting the electron output from the IFEL into another laser accelerator. These experiments used wigglers that provided a sinusoidal field variation, either as a circularly polarized- or dipole-type field, as did previous theory [3]. Results from experiment were interpreted by codes which also used sinusoidal field variation in the wiggler. Although the latter is a logical choice for the IFEL, and is teadily fabricated, it turns out that it is not the most effective way to use the magnetic field capabilities of the wiggler for electron acceleration; furthermore it is an approximation to the physics. In this paper we discuss another case, the square-wave wiggler-which, although non-physical, suggests a simple modification of present IFEL experiments which should yield improved performance $[1,7]$.
Of course, an actual wiggler cannot be truly sinusoidal[8], nor can a square-wave field variation be realized. However, it is possible to develop a strong nonlinearity of the sinusoidal pattern in a ferromagnetic wiggler by operating the device into the field regime where the material becomes saturated. The square wave pattem is therefore a limiting case [1]. On the other hand, the interpretation of daca from IFEL experiments using codes based on sinusoidal field patrerns may result in errors, depending on how closely the actual field pattern approximates a sine wave. In what follows, we present a theory $[1,2,7]$ and results from numerical simulation which models a non-physical square wave wiggler, and then generalize the treatment to include realizable sinusoidal wigglers afflicted with a high degree of non-sinusoidal nonlinearity [1]. In the limit of the square wave, we find an improvement in accelerating gradient by a factor of two.

\section{THEORY - SQUARE WAVE MODEL}

We consider the motion of an electron under the action of a linearly polarized laser electromagnetic field and an applied transverse magnetic fieid of the wiggler $(\mathrm{Bw})$ in the form of a square wave. The wave and the particles move in the z-direction, and the components of relativistic motion are:

$$
\left\{\begin{array}{l}
\frac{d}{d t}\left(m r v_{z}\right)=e \beta_{z} E_{=0} \sin \dot{0} \div e \beta_{z} B_{w} f(\dot{\phi}) \\
\frac{d}{d t}\left(r n \tau v_{z}\right)=e\left(1-\beta_{z}\right) E_{z=0} \sin \dot{\phi}-e \beta_{z} B_{w} f(\dot{0})
\end{array}\right.
$$

where Ex $=B y=-E x o \sin \phi, \phi=k(c t-z)$, the applied magnetic field of the wiggler is $B \circ f(\phi)$, and $f(\phi)$ Is a unit square wave that switches sign at odd numbers of $\pi / 2$ and $f(0)=-1$. Integrating (2), and taking $\Lambda_{w}$ to be the wiggler period,

$$
m \gamma v=\frac{e E_{z 0}}{k c} \cos \dot{\varphi}-\frac{e B_{z}}{c} \int_{0}^{*} d t v=f(\dot{\varphi})
$$




$$
\begin{aligned}
& \int_{0}^{x} d t v_{=}(\dot{\varphi})=\int_{0}^{x} d z f(\dot{\phi})=\frac{\phi \cdot D}{4} g(\dot{\varphi}) \text { and } g(\dot{\theta}) \\
& g(\dot{\phi})=-\frac{8}{\pi^{2}} \sum_{n=1}^{\infty} \frac{1}{n^{2}} \sin \frac{n \pi}{2} \sin n \dot{\phi} \\
& v_{z}=\frac{e E_{=0}}{k c} \cos \dot{\phi}-\frac{e B_{w} \Lambda_{\nu}}{4 m c^{2} \gamma} g(\dot{\phi}) \\
& m c^{2} \frac{d \gamma}{d t}=
\end{aligned}
$$

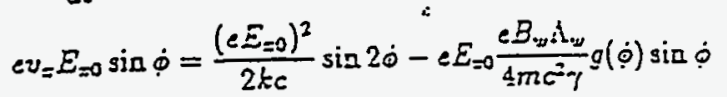

Neglecting the $\sin 2 \phi$ term in the energy equation, we find $d \gamma / d t \sim(2 / \pi)(\Lambda K / \gamma)$ with $K$ the wiggler parameter; which is about twice the result for a planar sinusoidal wiggler.

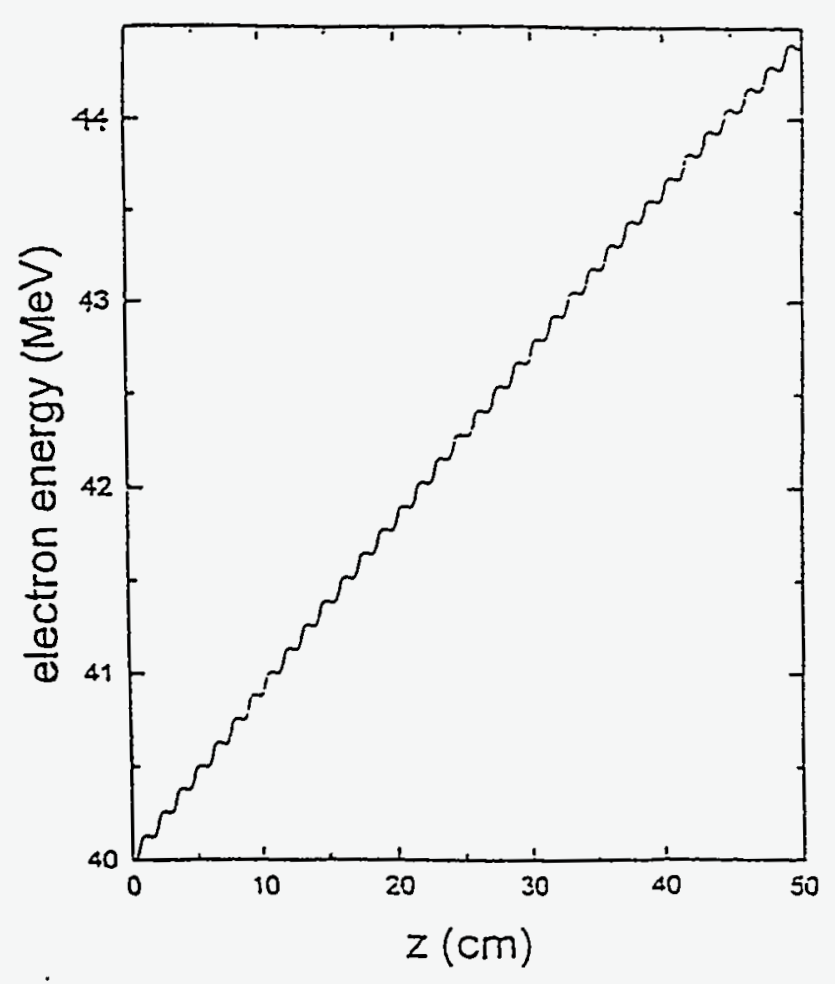

Figure la

In Figure la and Ib we show a computed example for the case of the recent Brookhaven experiment which reported $-2 \mathrm{MeV}$ increase on a $40 \mathrm{MeV}$ input beam due to the IFEL[5]. Taking their design (see Table I, loc. cit.), but with a square-wave wiggler, one finds in Figure la that the energy increase is about twice that obtained or expected in that experiment. The orbit of the electron in the transverse direction (Figure $1 b$ ) is stable, but nonsinusoidal: this results from the fact that $\mathrm{dPx} / \mathrm{dt}$ is a square wave, and $P x$ is therefore piecewise linear and $x(z)$ is piecewise quadratic. In fact, the reason that the squarewave wiggler performs better is that the electrons experience the high field values over a much larger fraction of their orbit, and so are accelerated more. Other simulations, taking more ambitious parameters for the wiggler and the

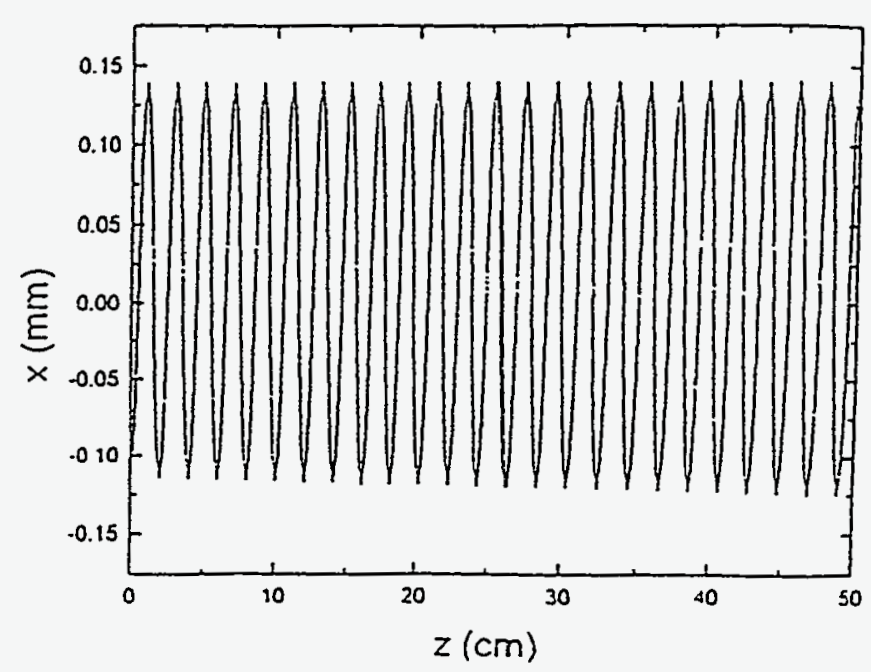

Figure $1 b$

laser, bear out this result. Since the IFEL accelerating gradient is almost linear in laser field, an improvement in gradient by two is equivalent to four times the laser power.

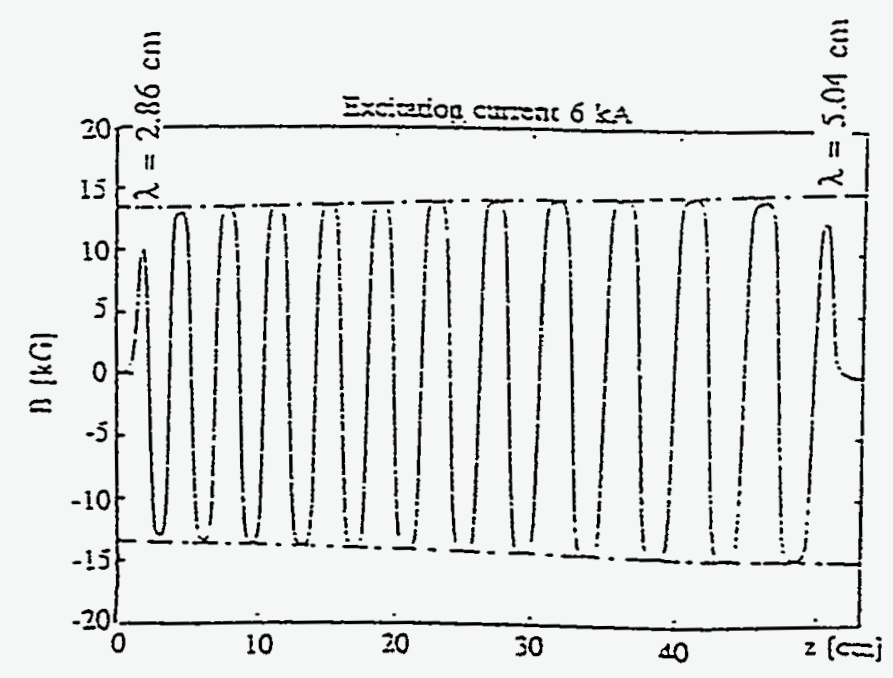

Figure 2: The Brookhaven fast excitation wiggler [9] 


\section{THEORY - REALIZABLE WIGGLER}

The present Brookhaven wiggler, which is made pardy of ferromagnetic material, will show pronounced nonsinusoidality if pushed to higher field values (see Figure 2 [9]) than the 10kG used in the past run [5]. Taking this as motivation to study a more realistic case than the square wave, the IFEL equations were cast in terms of a wiggler function that was modeled as a "multiple sine" function. One can consider a function

$$
\sin (\sin (\sin \ldots . . . . . .(\sin z)=y N(z)
$$

where the repeated sine operation introduces a nonsinusoidal flattening of the wiggler field, such as observed $[1,7]$.
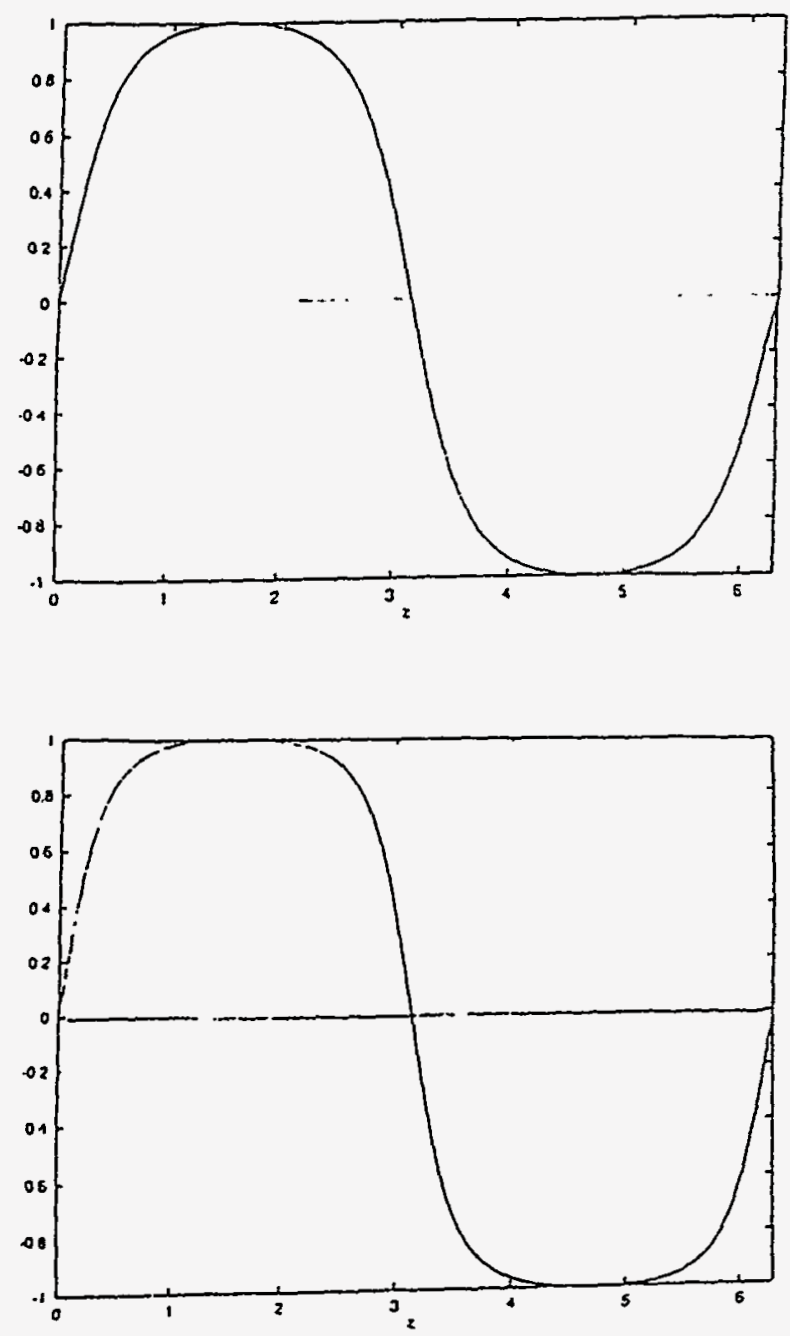

Figure 3 yN(z), multiple sine function of order $N$ : above, Fig. 3a, $N=10$; below, Fig. $3 b, N=20$.
In Figure 3a we show a case of a multiple-sine function of order ten, and in Figure $3 b$, of order 20. In the former case, the energy gain is enhanced by $40 \%$ above the energy gain of the sinusoidal wiggler (multiple sine of order one), and in the latter, by $60 \%$. Thus, we look forward to the operation of the IFEL in the regime where the ferromagnetic elements of a typical dipole wiggler become saturated, and the wiggler profile exhibits flattening. One might expect this to become possible with only minor changes in the wiggler design or construction, and therefore the improvement of energy gain is achieved in a painless way. If the departure from wiggler sinusoidality is not large, this study points out the need for careful characterization of the wiggler field so that accurate comparison with theory using codes that model the wiggler correctly can be made. In the present experiment [5], the wiggler effect may not stand out because the difficulty of measuring the $\mathrm{CO}_{2}$ laser power permits some latitude in interpreting the acceleration result.

\section{REFERENCES}

(1) Z.Parsa, "An Inverse FEl with a Square Wave Wiggler[IFELSW]". An Experimental Proposal to the ATF Steering Commitie (sec. 1-4). October 30. 1996 (ITP96-Report): Z. Parsa. "Square Wave Wiggler IFEL". (Sec. 5). Addition to proposal for IFELSW. November 201996 (ITP96-Report).

[2] Z. Parsa and M. Pato, -IFEL Acceleration with a Square-Wave Wiggler". conference proceedings "New Modes of Particle Acceleration: Techniques and Sources", AIP Press (1997)

[3] E.B. Courant . C. Pellegnini, and W. Zackowitz, Phys Rev. A32 2813 (1985)

[4] 1. Wemick and T.C. Marshall, Phys. Rev. A46, 3566 (1992)

[5] A. van Steenbergen, et al. Phys. Rev. Lett. 77, 2690 (1996)

[6] Y. Liu et al, paper $3^{\circ} \mathrm{C} .03$ of this meeting

[7] Z. Parsa, "Improved NAIBEA and IFEL", in abstracts (APS log no 5933) for the Joint April APS/APT meeting, Washington DC 1997

[8] S.Y. Park et al. J. Appl. Phys. 23 , 1320 (1982)

[9] CAP/ATF Meeting Report. December 1995 [Brookhaven] 